\title{
SARCOCYSTIS PANTHEROPHISI N. SP., FROM EASTERN RAT SNAKES (PANTHEROPHIS ALLEGHANIENSIS) AS DEFINITIVE HOSTS AND INTERFERON GAMMA GENE KNOCKOUT MICE AS EXPERIMENTAL INTERMEDIATE HOSTS
}

\author{
S. K. Verma, D. S. Lindsay*, J. D. Mowery†, B. M. Rosenthal, and J. P. Dubey \\ U.S. Department of Agriculture, Agricultural Research Service, Beltsville Agricultural Research Center, Animal Parasitic Diseases Laboratory, Building 1001, \\ Beltsville, Maryland 20705-2350. Correspondence should be sent to J. P. Dubey at: Jitender.Dubey@ars.usda.gov
}

\begin{abstract}
Here, we report a new species, Sarcocystis pantherophisi n. sp., with the Eastern rat snake (Pantherophis alleghaniensis) as natural definitive host and the interferon gamma gene knockout (KO) mouse as the experimental intermediate host. Sporocysts $(n=15)$ from intestinal contents of the snake were $10.8 \times 8.9 \mu$ m. Sporocysts were orally infective to KO mice but not to laboratory-raised albino outbred house mice (Mus musculus). The interferon gamma KO mice developed schizont-associated neurological signs, and schizonts were cultivated in vitro from the brain. Mature sarcocysts were found in skeletal muscles of KO mice examined 41 days postinoculation (PI). Sarcocysts were slender, up to $70 \mu \mathrm{m}$ wide and up to $3.5 \mathrm{~mm}$ long. By light microscopy, sarcocysts appeared thin-walled $(<1 \mu \mathrm{m})$ without projections. By transmission electron microscopy, the sarcocyst wall was a variant of "type 1" (type 1i, new designation). The parasitophorous vacuolar membrane (pvm) had approximately 100-nm-wide $\times 100$-nm-long bleb-like evaginations interspersed with 100-nm-wide $\times$ 650-nm-long elongated protrusions at irregular distances, and invaginations into the ground substance layer (gs) for a very short distance $(6 \mathrm{~nm})$. The gs was smooth, up to 500 $\mathrm{nm}$ thick, without tubules, and contained a few vesicles. Longitudinally cut bradyzoites at 54 days PI were bananashaped, $7.8 \times 2.2 \mu \mathrm{m}(\mathrm{n}=5)$. Molecular characterization using $18 S \mathrm{SRNA}, 28 S \mathrm{rRNA}, \mathrm{ITS}$ - 1 , and $\operatorname{cox} 1$ genes indicated a close relationship with other Sarcocystis parasites that have snake-rodent life cycles. The parasite in the present study was molecularly and biologically similar to a previously reported isolate (designated earlier as Sarcocystis sp. ex Pantherophis alleghaniensis) from P. alleghaniensis, and it was structurally different from other Sarcocystis species so far described.
\end{abstract}

Sarcocystis species have a 2-host life cycle, with herbivores as intermediate hosts and carnivores as definitive hosts (Dubey et al., 2016). Humans serve as both definitive and intermediate hosts. Clinically, sarcocystosis is typically a mild intestinal illness when humans are the definitive host. Muscle infections with sarcocysts when humans are the intermediate host are usually asymptomatic and are considered incidental findings. Recently, mysterious and diagnostically challenging outbreaks of human illness associated with muscular sarcocyst infection have been reported in persons that had a history of travel to Malaysia and surrounding regions. These human infections have been speculated to be caused by Sarcocystis species that have snakes as definitive hosts (reviewed by Dubey et al., 2016).

Little attention has been paid to Sarcocystis infection occurring in snakes in the United States. We isolated Sarcocystis sporocysts from the intestinal contents of a naturally infected Eastern rat snake (Pantherophis alleghaniensis) from Maryland (Verma et al., 2016). The sporocysts were infectious orally to interferon gamma gene knockout (KO) mice, but not outbred albino mice (Mus musculus). The KO mice died or became ill with neurological signs, but we could not previously find sarcocysts. In this report, we confirm these findings with another isolate from a $P$. alleghaniensis specimen and describe the occurrence of mature sarcocysts in KO mouse muscle. In this paper, we describe the

Received 3 January 2017; revised 7 June 2017; accepted 9 June 2017.

* Department of Biomedical Sciences and Pathobiology, College of Veterinary Medicine, Faculty of Health Sciences, Virginia Tech, Blacksburg, Virginia 24061.

$\dagger$ U.S. Department of Agriculture, Agricultural Research Service, Beltsville Agricultural Research Center, Electron and Confocal Microscopy Unit, Building 12, Beltsville, Maryland 20705.

DOI: $10.1645 / 17-2$ sarcocysts and propose a new species name: Sarcocystis pantherophisi n. sp.

\section{MATERIALS AND METHODS}

Naturally infected snake, in vivo and in vitro cultivation, and molecular investigations

A deceased adult Eastern rat snake (P. alleghaniensis) was found on the road of the Beltsville Agricultural Research Center, Maryland, in October 2015. The snake was evaluated for Sarcocystis infection. Sporocysts collected from the intestinal contents of this snake were bioassayed in laboratory-reared outbred Swiss Webster (SW, Mus musculus) and KO mice (Table I), schizogonic development was studied in cell culture, and DNA was extracted from sarcocysts characterized exactly as described by Verma et al. (2016) for our first isolate from P. alleghaniensis.

\section{Transmission electron microscopy (TEM)}

Skeletal muscles of 2 infected KO mice (nos. 650, 298; Table I) were fixed in $2.5 \%$ glutaraldehyde, $0.05 \mathrm{M} \mathrm{Na}$ cacodylate, and $0.005 \mathrm{M} \mathrm{CaCl}_{2}(\mathrm{pH} 7.0)$ and then refrigerated at 4 C. Samples were processed for TEM, and thin sections on grids were stained with $4 \%$ uranyl acetate and $3 \%$ lead citrate and imaged at $80 \mathrm{kV}$ with a Hitachi HT-7700 TEM (Hitachi High Technologies America Inc., Dallas, Texas).

\section{Phylogenetic analysis}

A phylogenetic tree based on $18 S$ rRNA sequences was reconstructed using the new sequence from Sarcocystis pantherophisi n. sp. Input sequences were the $18 S$ rRNA regions of different Sarcocystis species and related taxon retrieved from 
TABLE I. Bioassay of sporocysts collected from a naturally infected Eastern rat snake (Pantherophis alleghaniensis) and inoculated into laboratoryreared mice.

\begin{tabular}{ccccc}
\hline & & & \multicolumn{2}{c}{ Diagnosis } \\
\cline { 4 - 5 } Mouse type & Mouse no. & $\begin{array}{c}\text { Necropsy } \\
\text { (days PI) }\end{array}$ & Brain & Muscle \\
\hline \multirow{2}{*}{$\begin{array}{c}\text { Interferon gamma gene } \\
\text { knockout (KO) mouse }\end{array}$} & $297 \mathrm{KO}$ & 54 & Schizonts & Sarcocysts \\
& $298 \mathrm{KO}$ & 48 & Schizonts & Sarcocysts \\
Swiss Webster (SW) & $650 \mathrm{KO}$ & 41 & Schizonts & Sarcocysts \\
outbred mouse & $296 \mathrm{KO}$ & 48 & Schizonts & Sarcocysts \\
& $295 \mathrm{SW}$ & 73 & Negative & Negative \\
& $648 \mathrm{SW}$ & 73 & Negative & Negative \\
& $647 \mathrm{SW}$ & 73 & Negative & Negative \\
& & & &
\end{tabular}

NCBI GenBank. One-thousand bootstrap replicates of the phylogenetic relationships among these sequences were reconstructed using Geneious Tree builder under the criterion of the Tamura-Nei genetic distance model and neighbor-joining method as implemented in Geneious version 9.0.4 (Guindon et al., 2010). The final data set incorporated 49 sequences, and Besnoitia bennetti (AY665399) as an outgroup.

\section{RESULTS}

\section{Sporocysts}

Fully sporulated sporocysts were found in the intestinal contents of the Eastern rat snake. Sporocysts measured $17.3 \times$ $10.9 \mu \mathrm{m}(16.8-19.7 \times 9.8-11.9, \mathrm{n}=15)$. Each sporocyst contained 4 elongated sporozoites and a residual body (Fig. 1). A Stieda body was not present in the sporocysts. Oocysts/sporocysts were infective to inbred $\mathrm{BALB} / \mathrm{c}$-derived $\mathrm{KO}$ mice but not to outbred SW mice (Table I).

\section{Sarcocysts}

Microscopic sarcocysts were seen in the skeletal muscles of all 4 KO mice, but not in any SW mice (Table I). Only a few sarcocysts were detected in tongue, abdomen, limbs, and masseter muscles. None was found in the heart. Sarcocysts were microscopic, slender, up to $3.5 \mathrm{~mm}$ long and $70 \mu \mathrm{m}$ wide (Fig. 2A, B). In sections stained with hematoxylin and eosin (HE), the sarcocysts were septate, and the sarcocyst wall was smooth and $<1 \mu \mathrm{m}$ thick (Fig. 2C, D).

Two sarcocysts from abdominal muscle of KO mouse no. 298 were examined by TEM at 48 days postinoculation (PI). The sarcocyst wall had a wavy undulating parasitophorous vacuolar membrane (pvm); the undulations were at irregular distances (Fig. $3)$. The pvm was lined by a very thin ( $<5 \mathrm{~nm}$ thick) electron dense layer (edl) and a less lucent layer (elc) beneath it; the total thickness of both layers was $<100 \mathrm{~nm}$ thick (Fig. 3). Both edl layers appeared smooth, without dense granules. The pvm had villar protrusions (vp) that appeared as small blebs (100 nm wide $\times 100 \mathrm{~nm}$ long), interrupted by evaginated flattened areas, $100 \mathrm{~nm}$ wide $\times 650 \mathrm{~nm}$ long in size (Fig. 3). The pvm was also invaginated for a very short distance (up to $60 \mathrm{~nm}$ ) into the ground substance layer (gs); invaginations lacked edl at irregular distances and thus appeared as holes (Fig. 3C). The gs was up to $500 \mathrm{~nm}$ thick, was

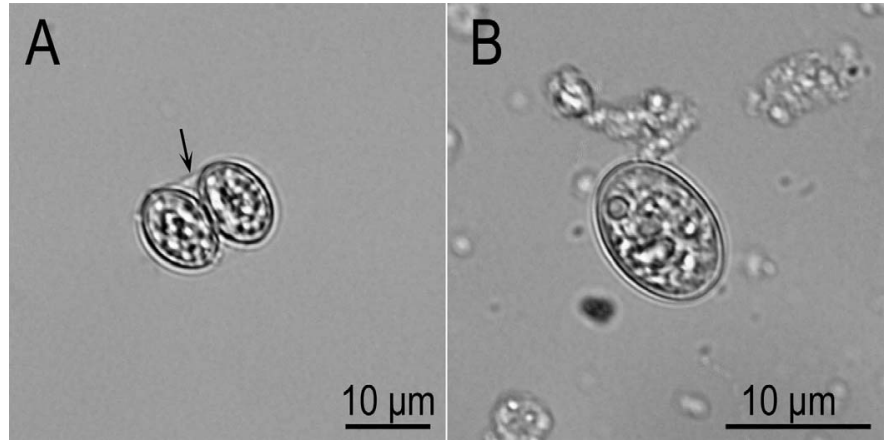

FIGURE 1. Oocyst and sporocyst of Sarcocystis pantherophisi n. sp. found in the intestinal contents of the Eastern rat snake, unstained. (A) Sporulated oocysts surrounded by a thin wall (arrow) and containing 2 sporocysts. (B) Sporocyst are ellipsoidal.

smooth, lacked microtubules, and contained a few vesicles (vs) (Fig. 3C).

Both metrocytes and bradyzoites were present in sarcocysts at 48 days PI (Fig. 2). Longitudinally cut bradyzoites at 54 days PI were $7.8 \times 2.2 \mu \mathrm{m}(7.1-8.4 \times 1.5-2.9 ; \mathrm{n}=5)$. Bradyzoites contained numerous conoid, irregularly arranged micronemes occupying the anterior end of the bradyzoite. No more than 2 rhoptries were seen in any bradyzoite section. There were several dense granules, amylopectin granules, and a subterminally located nucleus. The amylopectin granules were few and often located at the nonconoidal end. Both metrocytes and bradyzoites divided by endodyogeny.

\section{Schizonts}

The inoculated KO mice developed neurological signs, resulting in the need for euthanasia between 41 to 54 days PI. Sarcocystis schizonts and merozoites were found in the brains, particularly in the cerebellum (Fig. 4). Neither sarcocysts nor schizonts/ merozoites were detected in any SW mice euthanized 73 days PI (Table I).

Histological examination of $\mathrm{KO}$ mice revealed meningoencephalitis, characterized by infiltration of mononuclear cells around blood vessels, and focal necrosis of the neural tissue, particularly in the cerebellum. Immature and mature schizonts and free and intracellular merozoites were scattered in the brain. Isolated inflammatory foci contained degenerating and intact merozoites were found among neutrophils. The schizonts were up to $45 \mu \mathrm{m}$ and contained up to $12-\mu \mathrm{m}$-long merozoites (Fig. 4).

\section{In vitro cultivation}

The merozoites in the inocula were infectious for CV-1 cell cultures, and the stages observed were similar to those described from the first snake isolate that had been designated as Sarcocystis sp. ex Pantherophis alleghaniensis (Verma et al., 2016). Examination of a drop of the homogenate used to inoculate flasks was positive for merozoites; however, subsequent in vitro development was limited. Examination of inoculated cultures in $25 \mathrm{~cm}^{2}$ flasks demonstrated developmental stages near the host cell nucleus in cultures examined after 7-9 days. Developing schizonts varied in size, varying with the maturity of the nucleus. They increased in size as the nucleus developed numerous nucleoli and became lobed. Immature schizont stages 


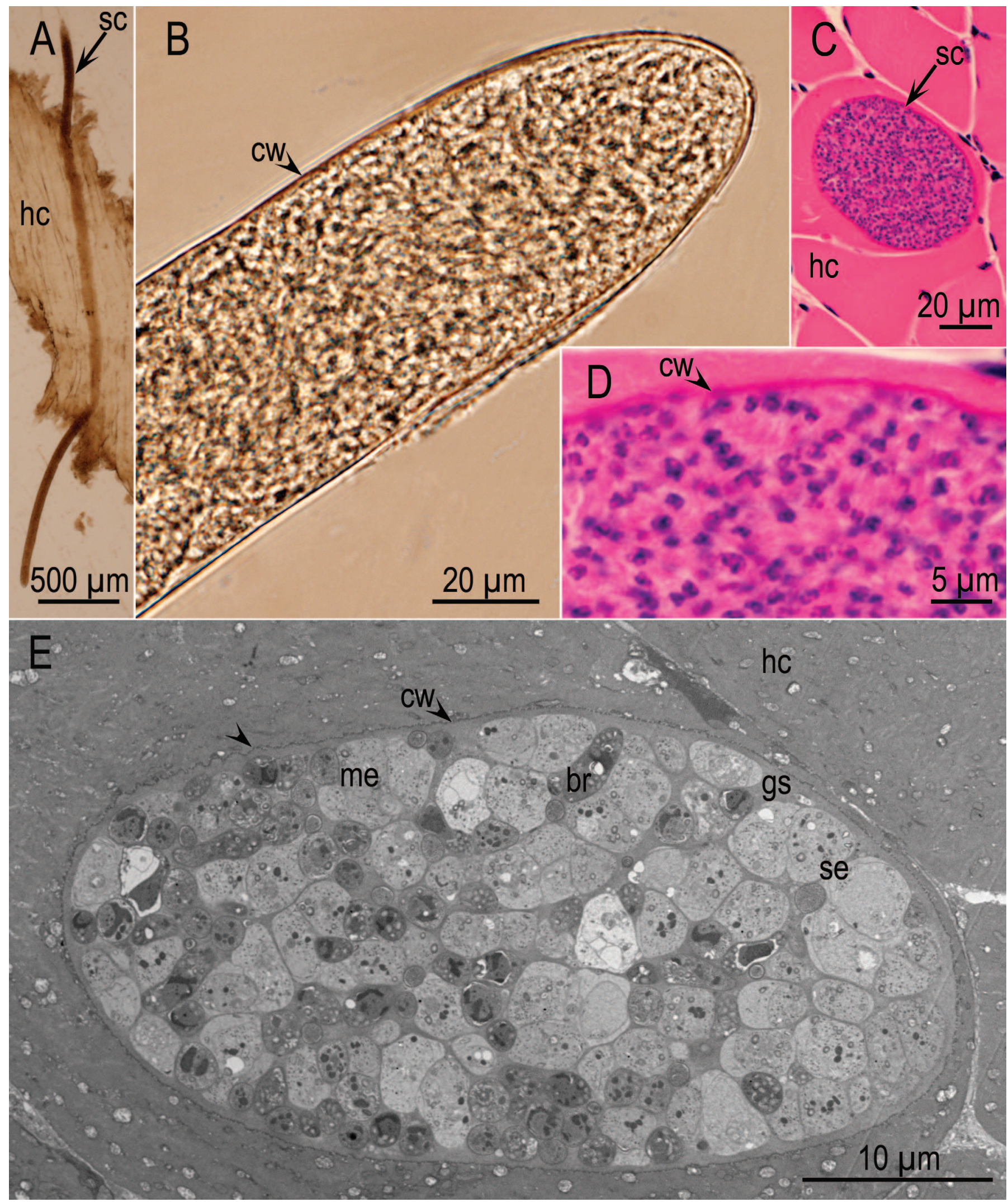

Figure 2. Sarcocysts of Sarcocystis pantherophisi n. sp., in muscles of experimentally infected KO mice, 48 days PI. Sarcocyst (sc), cyst wall (cw), host cell (hc). $\mathrm{A}$ and $\mathrm{B}=$ unstained, $\mathrm{C}$ and $\mathrm{D}=$ histological sections stained with hematoxylin and eosin, $\mathrm{E}=\mathrm{TEM}$. (A, B) Note slender sarcocysts, with no protrusions on cyst wall. (C, D) Note thin cyst wall (cw), without any visible protrusions. (E) The sarcocyst has undulating surface. Note variability in thickness (arrowheads) of the cyst wall (cw). The ground substance layer (gs) is smooth and continued in the interior of the sarcocyst as septa (se). Most organisms are metrocytes (me) with a few bradyzoites (br). 

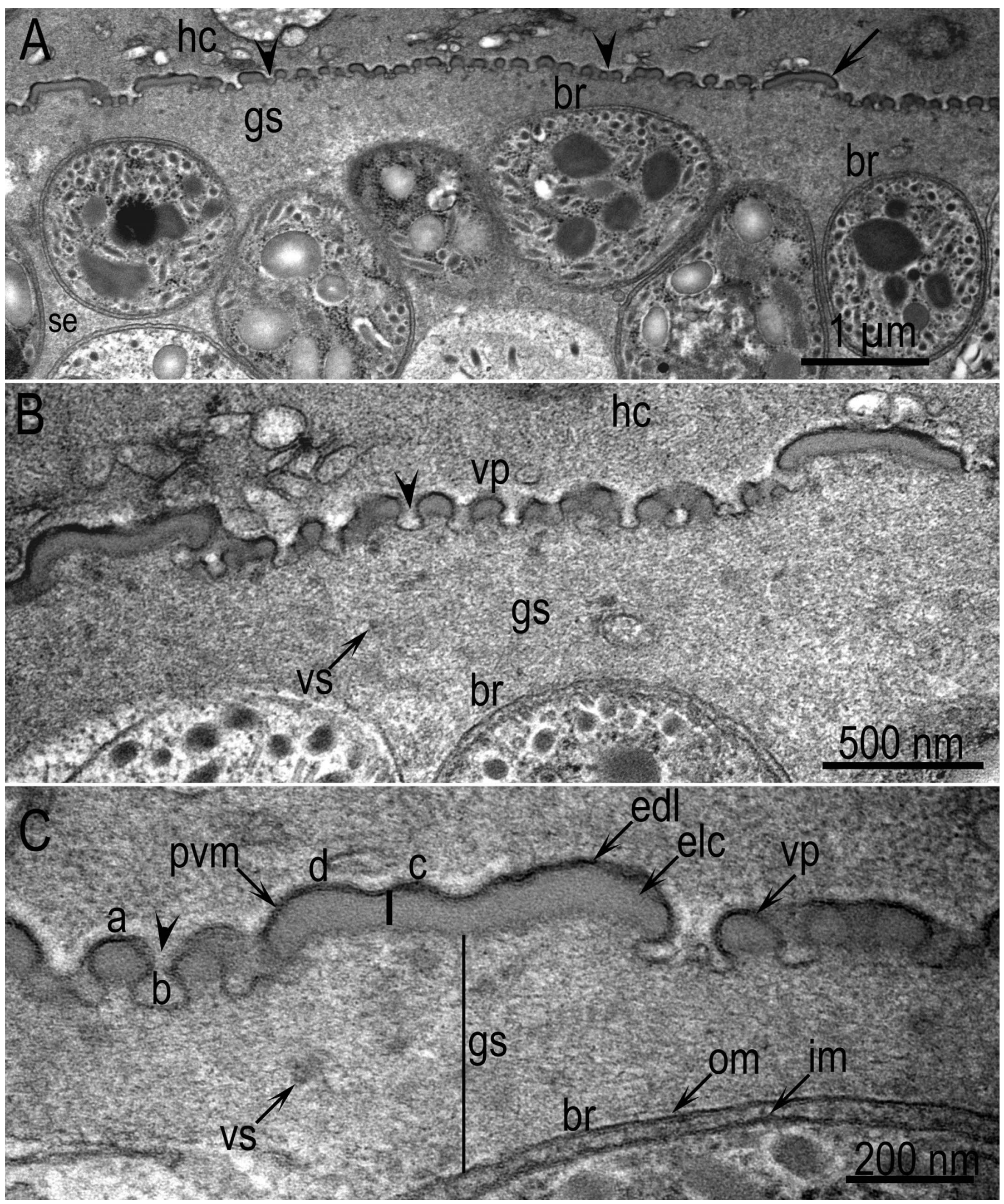

FIGURE 3. TEM of a Sarcocystis pantherophisi n. sp. sarcocyst in KO mouse, 48 days PI. (A) The sarcocyst has an undulating surface. Note variability in thickness (arrowheads) of the cyst wall (cw). Note ground substance layer (gs), septa (se), host cell (hc), villar protrusion (vp), metrocytes (me), and bradyzoites. (B, C) Higher magnification of cross section of a sarcocyst. Note parasitophorous vacuolar membrane (pvm) lined by electron dense layer (edl) and electron lucent layer (elc) of uneven thickness, almost missing in areas of invaginations of the pvm in the gs (arrowheads), and vesicles (vs). Also note the juxtaposition of a bradyzoite plasma lemma with outer (om) and inner membrane (im). 


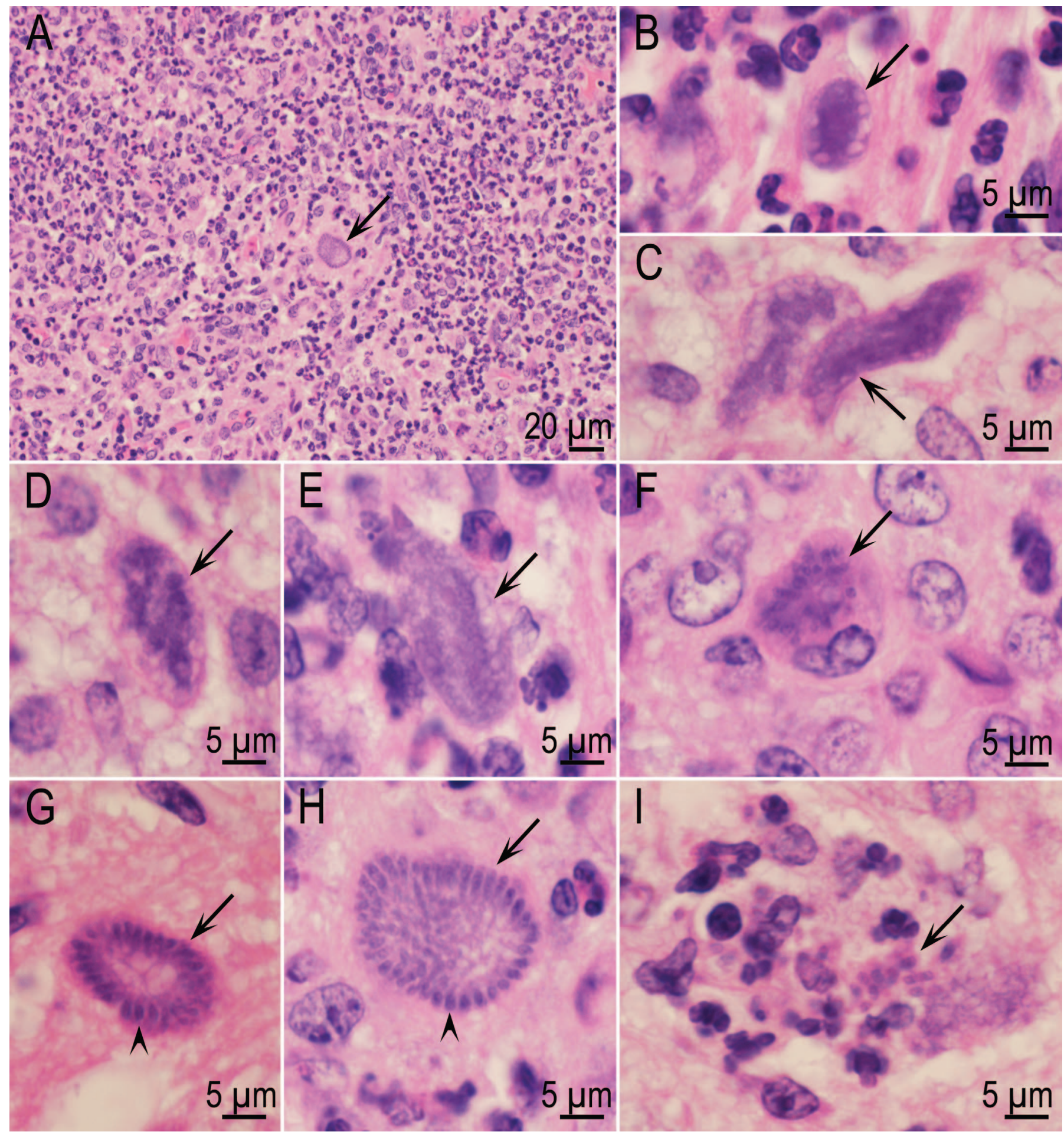

FIGURE 4. Sarcocystis pantherophisi $\mathrm{n}$. sp. schizogonic stages in the brain of experimentally infected KO mice inoculated with sporocysts, 48 days PI, hematoxylin and eosin stain. (A) A schizont (arrow) in an area of severe encephalitis. (B-F) Developing schizonts with lobulated nucleus with merozoites forming on surface (arrows). (G, H) Mature schizonts containing more than 50 merozoites arranged parallel to each other, forming rosette-like structures (arrows), and individual merozoites (arrowheads). (I) Focal encephalitis and necrosis around free merozoites (arrow). 


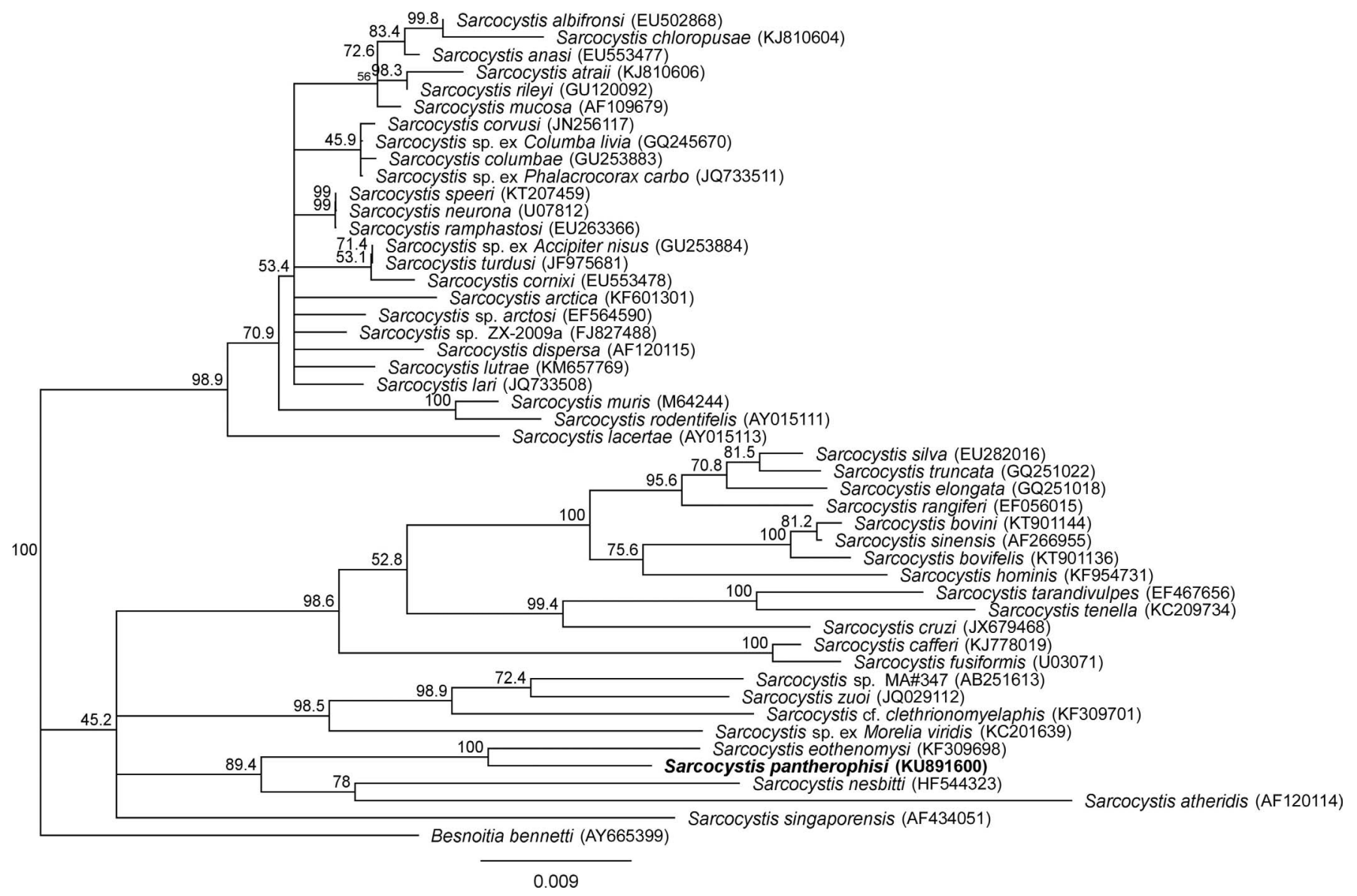

Figure 5. Phylogenetic tree based on $18 S r R N A$ sequences. Input sequences were the $18 S r R N A$ regions of various species of Sarcocystis retrieved from GenBank, and new $18 S$ rRNA sequence obtained from Sarcocystis pantherophisi $\mathrm{n}$. sp. Accession numbers of sequences follow the species name. One-thousand bootstrap replicates were performed using Geneious Tree builder using the neighbor-joining method based on Tamura-Nei distances, as implemented in Geneious version 9.0.4. Sarcocystis pantherophisi n. sp. showed close relationship with Sarcocystis eothenomysi (from a vole in China, and suspected to have a snake definitive host), Sarcocystis nesbitti (infections are documented in primates, including humans, but rodents are suspected as natural intermediate hosts and snakes as definitive hosts), and Sarcocystis atheridis (CD1 and Barbary striped mice as experimental intermediate hosts and Nitsche's bush viper, Atheris nitschei nitschei, as definitive host).

were $16-40 \times 9-30 \mu \mathrm{m}($ mean $=26.2 \times 17.0 \mu \mathrm{m} ; \mathrm{n}=5)$. The largest multilobed schizont observed was $54 \times 26 \mu \mathrm{m}$. Mature schizonts could contain more than 50 merozoites. Merozoites were produced on blastophores that developed on the surface of schizonts. Cultures were discarded after 2 mo of culture.

\section{PCR and DNA analysis}

PCR analysis with sarcocyst DNA as the template yielded amplicons of the expected size for the $18 S$ rRNA (in 3 fragments, $\sim 1,800 \mathrm{bp}$ ), $28 S \mathrm{rRNA}$ (in 2 fragments, $\sim 1,500 \mathrm{bp}$ ), ITS-1 ( 900 bp), and coxl $(\sim 1,050$ bp) loci. PCR-DNA sequencing of amplicons resulted in unambiguous sequences of 3 nuclear DNA regions: $18 S \operatorname{rRNA}, 28 S \mathrm{rRNA}$, and ITS-1, and a mitochondrial DNA locus, coxl. These sequences matched $100 \%$ with Sarcocystis species reported from the first snake (Verma et al., 2016), with accession numbers KU891600 (18S rRNA), KU891601 (28S rRNA), KU891602 (ITS-1), and KU891603 (coxl) and are designated as originating from Sarcocystis pantherophisi $\mathrm{n}$. sp.

In the phylogenetic tree based on $18 S$ rRNA sequences, $S$. pantherophisi n. sp. Sequences clustered consistently with Sarco- cystis eothenomysi (from a vole in China, and suspected to have a snake definitive host; $\mathrm{Hu}$ et al., 2014) and, with less bootstrap support, Sarcocystis nesbitti (nonhuman primates as intermediate hosts and humans as aberrant hosts; rodents have been hypothesized as natural intermediate hosts and snakes as natural definitive hosts; Dubey et al., 2015), and Sarcocystis atheridis (Fig. 5). The S. atheridis sporocysts were isolated from a Nitsche's bush viper (Atheris nitschei nitschei), from Uganda, and experimental transmission resulted in sarcocyst development in the skeletal muscles of laboratory mice (CD1 and Barbary striped mice [Lemniscomys barbarus]) but not in rats (Wistar H) (Dolezel et al., 1999; Slapeta et al., 1999).

\section{DESCRIPTION}

\section{Sarcocystis pantherophisi $\mathbf{n} . \mathbf{s p}$.}

(Figs. 1-5)

Diagnosis: Sporocysts $10.8 \times 8.9 \mu \mathrm{m}$ with 4 sporozoites. Schizonts in brain of KO mice. Sarcocysts in skeletal muscle of KO mice. Sarcocyst wall $<1 \mu \mathrm{m}$ thick, smooth with "type 1i" wall. Bradyzoites up to $7.8 \mu \mathrm{m}$ long. 


\section{Taxonomic summary}

Type definitive host: Eastern rat snake (P. alleghaniensis).

Natural intermediate host: Unknown.

Experimental intermediate host: Interferon gamma gene knockout mouse.

Type locality: Beltsville, Prince George's County, Maryland, United States.

Other localities: Unknown.

Etymology: Species named after the genus of the definitive host Eastern rat snake (P. alleghaniensis).

Specimens deposited: Hematoxylin and eosin-stained histologic slides from the KO mouse: no. D10001-1, 48 days PI (containing sarcocysts in skeletal muscle-USNM1422356), and D 88783, 54 days PI (containing schizonts in brain, USNM1422360), have been catalogued at the National Parasite Collection housed at the Smithsonian Institution, National Museum of Natural History, Department of Invertebrate Zoology (USNM).

Sequences deposited: Sequences deposited in NCBI GenBank with accession numbers KU891600 (18S rRNA), KU891601 (28S rRNA), KU891602 (ITS-1), and KU891603 (coxl).

\section{Remarks}

Sarcocystis species are generally host-specific for the intermediate host, especially those using rodents as intermediate hosts (Dubey et al., 2016). The ultrastructure of the sarcocyst wall is a useful taxonomic criterion for differentiating Sarcocystis species within a given host. The sarcocyst wall of the species in the present study is structurally distinct from other species described. Dubey et al. (2016) proposed 42 types of sarcocyst walls with many subdivisions within some of the wall types. By light microscopy, sarcocysts were grouped as thin-walled $(<1 \mu \mathrm{m})$ or thick-walled $(>2 \mu \mathrm{m})$. The "type 1" sarcocyst wall is thin and has small blebs on the wall; it was subdivided into 8 "subtypes 1a-1h" depending on the villar protrusions (Dubey et al., 2016). To this description, we have added a new "type 1i" for the Sarcocystis wall in the present study. The new "type $1 \mathrm{i}$ " is defined as follows: The sarcocyst wall had a wavy undulating parasitophorous vacuolar membrane (pvm); the undulations were at irregular distances. The pvm was lined by a very thin $(<5 \mathrm{~nm}$ thick $)$ electron dense layer and a less lucent layer beneath it; the total thickness of both layers was $<100 \mathrm{~nm}$ thick (Fig. 3). The pvm had villar protrusions (vp) that appeared as small blebs (100 $\mathrm{nm}$ wide $\times 100 \mathrm{~nm}$ long), interrupted by evaginated flattened areas, $100 \mathrm{~nm}$ wide $\times 650 \mathrm{~nm}$ long in size (Fig. 3 ). The pvm was also invaginated but only for a very short distance (up to $60 \mathrm{~nm}$ ) into the gs; invaginations lacked edl at irregular distances and thus appeared as holes. The gs was up to $500 \mathrm{~nm}$ thick, was smooth, lacked microtubules, and contained a few vesicles.

Of the 17 species of Sarcocystis with snakes as definitive hosts, sarcocysts of 12 are thick-walled (Sarcocystis acanthocolubri with lizards as intermediate host $[\mathrm{IH}]$ and colubrid snakes as definitive host [DH]; Sarcocystis gongyli with skinks as IH and diadem snakes as DH; Sarcocystis lacerate with lizard as IH and smooth snake as DH; Sarcocystis podarcicolubris with lizard as IH and whip snakes as DH; Sarcocystis stenodactylicolubris with gecko as IH and Dahl's whip snake as DH; Sarcocystis cletherionomyelaphis with voles as IH and Aesculapian snake as DH; Sarcocystis hoarensis with rats and gerbils as $\mathrm{IH}$ and Bitis snakes as $\mathrm{DH}$; Sarcocystis murinotechis with Norway rat as IH and tiger snake as
DH; Sarcocystis muriviperae with house mouse as IH and Palestinian viper as DH; Sarcocystis singaporensis with Norway rat as IH and python as DH; Sarcocystis villivillosi with Norway rat as IH and python as DH; Sarcocystis zamani with Norway rat as IH and python as DH; and Sarcocystis zoui with Norway rat as IH and King snake as DH) (reviewed in Dubey et al. 2016). Sarcocysts of 5 species with snakes as DH have thin walls. A detailed comparison of the TEM of these species indicated that the Sarcocystis pantherophisi $\mathrm{n}$. sp. proposed here has a distinct sarcocyst wall, designated "type 1i."

Biologically, 3 of these (Sarcocystis schneideri, Sarcocystis bunopusi, Sarcocystis chalcidicolubris) use lizards or skinks as IH. Two of these (Sarcocystis atheridis and Sarcocystis crotali) use house mice as IH. Two of these (Sarcocystis dirumpens and Sarcocystis gerbilliechis) use Mastomys natalensis and gerbils as their IH. Molecularly, S. pantherophisi n. sp. shows close similarities with Sarcocystis eothemomysi (large oriental vole, Eothenomys miletus, as IH and venomous snake hypothesized as DH; have irregular projections, have "type 18" sarcocyst wall). Sarcocystis nesbitti (nonhuman primates as IH and human as aberrant hosts, rodents hypothesized as $\mathrm{IH}$ and snake as $\mathrm{DH}$; have knob-like elevations similar to the "type 1a" sarcocyst wall), Sarcocystis zuoi (Norway rats-king rat-snake cycle, long, have finger-like protrusions with a highly branched base), Sarcocystis sp. ex Morelia viridis GM (green pythons, Morelia viridis, as DH), Sarcocystis singaporensis (Norway rats as IH and Python reticulatus as $\mathrm{DH}$; have spatula- or sausage-like protrusions in sarcocyst wall), and many other species of Sarcocystis employ snakes (python, cobra, rat snake, viper) as their DH.

\section{DISCUSSION}

The Eastern rat snake ( $P$. alleghaniensis) is a species of nonvenomous, colubrid snake, endemic to North America and Central America. Its diet consists primarily of small rodents, frogs, toads, lizards, eggs, and young birds. The parasite found in the present study closely resembles a Sarcocystis species (Sarcocystis montanaensis-like) that cycles between the speckled kingsnake (Lampropeltis getula holbrooki) and prairie vole (Microtus ochrogaster). Sarcocystis montanaensis was named for thin-walled sarcocysts found in muscles of 3 of 31 meadow voles (Microtus pennsylvanicus) necropsied in 1980 (Dubey, 1983). Ultrastructurally, the sarcocyst wall had minute knob-like projections on the sarcocyst wall. Lindsay et al. (1992) found a similar parasite in a naturally infected speckled kingsnake in 1989 from Arkansas. Sporocysts isolated from the snake were infectious to the prairie voles but not infectious to laboratory mice (Mus musculus) or white-footed mice (Peromyscus leucopus) (Lindsay et al., 1992). Schizonts were detected in livers of the voles examined 5, 7, and 8 days PI. Mature sarcocysts were seen in both voles examined 117 days PI (Lindsay et al., 1992). Sarcocysts observed in histological sections were microscopic, with a width of $45-84 \mu \mathrm{m}$; the length of the sarcocyst was not stated. All sarcocysts were observed in the tongue, with the exception of 1 cyst in the diaphragm. Ultrastructurally, the pvm had knob-like protrusions and protrusions that were considered to be flattened due to plane of section (Lindsay et al., 1992). The parasite was thought to be $S$. montanaensis, based on knob-like protrusions. Molecular data of this species are not available. Retrospectively, it appears that the sarcocysts induced in the prairie vole were 
different from the $S$. montanaensis sarcocysts from the meadow vole. Sarcocysts in the meadow vole were grossly visible, rice grain-like structures, and the sarcocyst wall lacked the flattened villar protrusions now described in detail in the present paper. Unlike the parasite in the speckled kingsnake, where schizonts were in livers of the voles, the present parasite's schizogony occurred in the brain of KO mice. Collectively, the data support a new name, Sarcocystis pantherophisi n. sp., for the species in the Eastern rat snake.

The $\mathrm{KO}$ mice have proven to be very useful in understanding the biology of Sarcocystis neurona, which causes fatal encephalomyelitis in horses (Equus caballus) (called EPM), and many other hosts, and is a major cause of mortality in sea otters (Enhydra spp.) (Dubey et al., 2015). The encephalitis in KO mice mimics the natural disease. The $\mathrm{KO}$ mice are considered an aberrant host for $S$. neurona because only schizonts are produced; sarcocysts are absent. In this respect, finding of sarcocysts in $\mathrm{KO}$ mice orally inoculated with sporocysts from snake feces is noteworthy and might assist in finding the natural intermediate host for $S$. pantherophisi n. sp.

\section{Compliance with ethical standards}

All investigations reported here were approved by the Institutional Animal Care and Use Protocol Committee of the U.S. Department of Agriculture (USDA). Mention of trade names or commercial products in this publication is solely for the purpose of providing specific information and does not imply recommendation or endorsement by the USDA; USDA is an equal opportunity provider and employer.

\section{LITERATURE CITED}

Dolezel, D., B. Koudela, M. Jirků, V. Hypsa, M. Obornik, J. VotÝPKA, D. Modrý, J. R. Slapeta, and J Lukes. 1999. Phylogenetic analysis of Sarcocystis spp. of mammals and reptiles supports the coevolution of Sarcocystis spp. with their final hosts. International Journal for Parasitology. 29: 795-798.
Dubey, J. P. 1983. Sarcocystis montanaensis and S. microti sp. $\mathrm{n}$. from the meadow vole (Microtus pennsylvanicus). Proceedings of the Helminthological Society of Washington 50: 318-324.

Dubey, J. P., R. Calero-Bernal, B. M. Rosenthal, C. A. Speer, AND R. FAYER. 2016. Sarcocystosis of animals and humans, 2nd ed. CRC Press, Boca Raton, Florida, 481 p.

Dubey, J. P., D. K. Howe, M. Furr, W. J. Saville, A. E. Marsh, S. M. Reed, And M. E. Grigg. 2015. An update on Sarcocystis neurona infections in animals and equine protozoal myeloencephalitis (EPM). Veterinary Parasitology 209: $1-42$.

Guindon, S., J. F. Dufayard, V. Lefort, M. Anisimova, W. HordiJk, AND O. Gascuel. 2010. New algorithms and methods to estimate maximum-likelihood phylogenies: Assessing the performance of PhyML 3.0. Systematic Biology 59: $307-321$

Hu, J. J, Q. Liu, Y. F. YAnG, G. W. Esch, Y. M. Guo, And F. C. Zou. 2014. Sarcocystis eothenomysi n. sp. (Apicomplexa: Sarcocystidae) from the large oriental vole Eothenomys miletus (Thomas) (Cricetidae: Microtinae) from Anning, China. Systematic Parasitology 89: 73-81

Lindsay, D. S., S. J. Upton, B. L. Blagburn, M. ToivioKinnucan, J. P. Dubey, C. T. McAllister, and S. E. Trauth. 1992. Demonstration that Sarcocystis montanaensis has a speckled kingsnake-prairie vole life cycle. Journal of the Helminthological Society of Washington 59: 9-15.

Slapeta, J. R., D. Modrý, And B. Koudela. 1999. Sarcocystis atheridis sp. nov., a new sarcosporidian coccidium from Nitsche's bush viper, Atheris nitschei Tornier, 1902, from Uganda. Parasitology Research 85: 758-764.

Verma, S. K., D. S. Lindsay, B. M. Rosenthal, and J. P. Dubey. 2016. Ancient, globally distributed lineage of Sarcocystis from sporocysts of the Eastern rat snake (Pantherophis alleghaniensis) and its relation to neurological sequalae in intermediate hosts. Parasitology Research 115: 2697-2704. 